\section{Avaliação da densidade mineral óssea em adolescentes do sexo feminino com transtorno alimentar}

\author{
Evaluation of bone mineral density in female \\ adolescents with eating disorders
}

Mariana Moraes Xavier da Silva', Durval Damiani', Louise Cominato'
1 Departamento de Endocrinologia Pediátrica, Instituto da Criança (ICr), Faculdade de Medicina da Universidade de São Paulo (FMUSP), São Paulo, SP, Brasil
Correspondência para: Mariana Moraes Xavier da Silva Rua João Huss, 405, ap. 1402 8605-490 - Londrina, PR, Brasil marianamxs@yahoo.com.br

Recebido em 13/Out/2012 Aceito em 4/Mar/2013

\section{INTRODUÇÃO}

$\mathrm{O}$ $s$ transtornos alimentares (TA) são quadros psiquiátricos que afetam principalmente adolescentes e adultos jovens, especialmente do sexo feminino, levando a grandes prejuízos biopsicossociais, com elevada morbidade e mortalidade (1).

A anorexia nervosa (AN) e o transtorno alimentar não especificado (TANE) são os TA mais frequentes na adolescência (2).

Segundo o Diagnostic and Statistical Manual of Mental Disorders (DSM-IV), a AN caracteriza-se por: restrição da ingestão alimentar com importante perda de peso, recusa em manter peso mínimo adequado para idade e altura, medo intenso em ganhar peso, distúrbio da imagem corporal e amenorreia (3).

A prevalência de AN em adolescentes do sexo feminino para apresentações que satisfazem todos os critérios é de $0,5 \%$. É a terceira doença crônica mais comum em meninas nessa faixa etária (4).

Indivíduos que não apresentam todos os critérios diagnósticos para o transtorno, isto é, com diagnóstico de TANE, são encontrados com maior frequência (5).

O TANE pode ser caracterizado pelos seguintes critérios: existem todos os sinais e sintomas de anorexia, 
porém a paciente apresenta ciclos menstruais regulares; há perda de peso significante, no entanto a paciente mantém IMC normal; a amenorreia ou o temor de engordar podem estar ausentes mesmo na presença de perda de peso marcante e de comportamentos para a redução de peso (3). O TANE corresponde a 40\%-60\% dos casos de TA em crianças (6).

A AN é responsável por anormalidades na mineralização óssea, que são bem conhecidas e descritas em mulheres adultas, porém menos documentadas em adolescentes. Está associada à diminuição da densidade mineral óssea (DMO), comprometendo o pico de massa óssea e aumentando o risco de fraturas (7).

A adolescência é o período crítico na vida de mulheres jovens para aquisição mineral óssea e obtenção do pico de massa óssea. Déficits importantes na massa óssea ocorrem como uma complicação precoce e frequente da AN na adolescência (8).

O diagnóstico de redução da massa óssea é feito por meio da densitometria óssea (DO) obtida por absorciometria com raios X de dupla energia (DXA), técnica eficaz e considerada como padrão-ouro no diagnóstico de densidade mineral óssea (DMO) $(9,10)$.

Para avaliar a DMO e diagnosticar redução da massa óssea em crianças e adolescentes, é utilizado o escore Z $(10,11)$.

Os valores são considerados anormais quando o escore $\mathrm{Z}$ é inferior a -2 (11).

A DO lombar é considerada mais sensível como medida de perda óssea trabecular nessa doença (12).

Mulheres jovens anoréxicas podem nunca atingir um pico adequado de massa óssea. Duas características clínicas da AN - deficiência estrogênica e perda de peso - são fatores de risco importantes para redução da massa óssea. É incerto se ocorre declínio progressivo e permanente na massa óssea dessas pacientes $(13,14)$.

O objetivo deste estudo é avaliar a DMO lombar (Ll-L4) em adolescentes do sexo feminino com transtorno alimentar no momento do diagnóstico e a evolução após seis meses e um ano de tratamento.

\section{SUBJEITOS E MÉTODOS}

Avaliação prospectiva de 35 adolescentes de 10 a 18 anos de idade, do sexo feminino, com transtorno alimentar, acompanhadas no ambulatório do PROTAD (Programa de Atendimento, Ensino e Pesquisa em Transtornos Alimentares na Infância e na Adolescência - Ambulatório de Bulimia e Transtornos Alimentares) do Instituto de Psiquiatria do Hospital das Clínicas da Faculdade de Medicina da Universidade de São Paulo, no período de abril de 2006 a dezembro de 2008 . As pacientes foram classificadas como AN ou TANE de acordo com os critérios diagnósticos do DSM-IV (3) e avaliadas por um psiquiatra infantil.

Critérios de inclusão: sexo feminino, idade entre 10 e 18 anos, ter diagnóstico de AN ou TANE. Foram excluídos pacientes com diagnóstico de outros transtornos alimentares como bulimia nervosa, portadores de doenças endocrinológicas concomitantes, em uso de anticoncepcional hormonal e/ou uso de corticoterapia e pacientes que não seguiram o cronograma do estudo.

As pacientes foram submetidas a tratamento psicológico, acompanhamento psiquiátrico e endocrinológico e terapia nutricional. $\mathrm{O}$ tratamento visa à recuperação nutricional e da menarca nas meninas com amenorreia primária ou a restauração da função menstrual nas com amenorreia secundária, além do controle ou remissão dos outros sintomas listados nos critérios do DSM-IV. Tratamento medicamentoso foi indicado apenas para outros transtornos psiquiátricos concomitantes ao transtorno alimentar como depressão, transtorno-obsessivo compulsivo e transtorno de ansiedade.

Todos os responsáveis pelas pacientes assinaram termo de consentimento livre e esclarecido para realização dos exames, e o estudo transcorreu de acordo com as normas estabelecidas pela Comissão de Ética para Análise de Projetos de Pesquisa do HC-FMUSP.

\section{Medidas antropométricas}

Peso: em quilogramas, aferido semanalmente por nutricionista da equipe multidisciplinar do PROTAD. Pacientes pesadas sem roupas em balança mecânica antropométrica Welmy $110 \mathrm{CH}$ com capacidade para $180 \mathrm{~kg}$ e divisões de $100 \mathrm{~g}$.

Altura: medida mensalmente por endocrinologista pediátrico da equipe multidisciplinar do PROTAD. Altura aferida em metros, em estadiômetro fixo à parede com escala em milímetros Tonelli E120P (padrão nacional), com amplitude de medida de $50 \mathrm{~cm}$ a $2,20 \mathrm{~m}$.

IMC: calculado semanalmente como peso em quilogramas dividido pela altura em metros ao quadrado $(\mathrm{kg} /$ $\mathrm{m}^{2}$ ). Foram utilizadas como referência as curvas de peso, estatura e IMC do National Center for Health Statistics/ Centers for Disease Control (NCHS/CDC) $2000(15,16)$.

Avaliação clínica: anamnese, exame físico geral e avaliação do estádio puberal de Tanner por endocrino- 
logista pediátrico da equipe multidisciplinar do PROTAD no início do acompanhamento, após seis meses e um ano do início do tratamento.

Avaliação laboratorial: exames laboratoriais (cálcio, fósforo, fosfatase alcalina) foram colhidos em três ocasiões (início, após seis meses e após um ano de tratamento) e nenhuma paciente apresentou alterações nesses parâmetros.

Idade óssea: avaliação da idade óssea por meio de radiografia de mãos e punhos pelo método de Greulich e Pyle (17). Foi realizada no início do acompanhamento e após um ano de tratamento. O laudo radiológico foi feito pelo mesmo radiologista. A idade óssea foi avaliada pelo método de Greulich e Pyle, pois não tivemos acesso às radiografias para comparação por métodos mais sensíveis tais como o TW3-RUS (18).

Densitometria óssea: foi realizada no início do acompanhamento, após seis meses e um ano do início do tratamento. A avaliação densitométrica da coluna lombar L1-L4 pelo método de DXA (absorciometria por dupla emissão de raios X) foi feita pelo aparelho Hologic QDR-4500A. Valores da DMO expressos em $\mathrm{g} / \mathrm{cm}^{2}$. O escore $\mathrm{Z}$ foi calculado em relação a indivíduos da mesma idade, sexo, raça e peso da paciente. Todos os exames foram feitos no mesmo aparelho e os laudos densitométricos foram avaliados pelo mesmo radiologista.

\section{Análise estatística}

$\mathrm{Na}$ análise estatística utilizou-se o programa SPSS 14. Estatística descritiva (medidas antropométricas, porcentagens, médias e desvios-padrão) foi utilizada para caracterizar a amostra. Usou-se o módulo nutricional do Epi-Info 2002 para determinar os percentis de IMC para idade e sexo.

A análise estatística foi feita usando o teste $t$ de Student para comparar variáveis contínuas. No estudo da evolução da idade óssea e densitometria óssea no tempo (iniciais e durante o seguimento das pacientes) e comparação entre os grupos TANE e AN, foi utilizada análise de variância (ANOVA) para medidas repetidas. $\mathrm{O}$ nível de significância foi determinado com valor de $\mathrm{p}$ $<0,05$.

\section{RESULTADOS}

Das 35 pacientes avaliadas, 60\% apresentavam AN e $40 \%$, TANE. A média de idade ao início do acompanhamento foi de $14,63 \pm 1,69$ anos (Tabela 1 ).
Tabela 1. Características clínicas e dados de internação de um grupo de 35 pacientes com AN ou TANE acompanhadas no PROTAD

\begin{tabular}{lc}
\hline AN, \% & 60 (21 pacientes) \\
TANE, \% & 40 (14 pacientes) \\
Idade, anos & $14,63 \pm 1,69$ \\
Idade da menarca, anos $(n=30)$ & $11,76 \pm 1,17$ \\
Duração da doença (pré-tratamento), meses & $13,51 \pm 13,07$ \\
Amenorreia primária, \% & 20 (5 pacientes) \\
Amenorreia secundária, \% & 80 (20 pacientes) \\
Tempo de amenorreia, meses & $9,75 \pm 8,39$ \\
Internação, \% & $48,6(17$ pacientes) \\
Tempo de internação, meses & $3,12 \pm 1,18$ \\
\hline
\end{tabular}

Todas as pacientes eram púberes no início do acompanhamento, e 30 pacientes $(85,7 \%)$ já haviam apresentado a menarca, com média de idade da menarca de $11,76 \pm$ 1,17 anos. Entre as pacientes, $71,4 \%$ apresentavam amenorreia, sendo $20 \%$ primária. O tempo médio de amenorreia secundária foi de $9,75 \pm 8,4$ meses, com tempo igual ou inferior a seis meses em $60 \%$ dos casos (Tabela 1 ).

Dos casos de amenorreia primária, $60 \%$ não apresentaram menarca ao longo de um ano de acompanhamento. Dos casos de amenorreia secundária, $50 \%$ voltaram a menstruar regularmente nos primeiros seis meses de tratamento, $20 \%$ entre seis meses e um ano e $20 \%$ persistiram amenorreicas.

O tempo de duração da doença pré-tratamento foi de $13,51 \pm 13,07$ meses. Em 37,1\% dos casos o período pré-tratamento foi igual ou inferior a seis meses e em $14,3 \%$ dos casos o tempo foi igual ou superior a dois anos. Das 35 pacientes, $17(48,6 \%)$ necessitaram de internação, com tempo médio de internação de $3,12 \pm 1,18$ meses (Tabela 1). Os principais motivos das internações foram desnutrição proteico-energética grave com caquexia, distúrbios hidroeletrolíticos, especialmente hipocalemia e piora dos sintomas anoréxicos, como recusa alimentar.

Como o estudo envolveu pacientes de 10 a 18 anos, algumas adolescentes estavam em fase de crescimento e estavam em fases diferentes de desenvolvimento puberal.

A média do escore $\mathrm{Z}$ de peso no início do tratamento foi de $-1,2(-1,85-0,59)$, após seis meses de tratamento, de $-0,64(-1,29-0,02)$ e após um ano de tratamento, de -0,56 (-1,24-0,07). A comparação da média do escore $\mathrm{Z}$ de peso inicial e após seis meses, inicial e após um ano de tratamento e a média aos seis meses comparada a um ano foram todas significantes ( $\mathrm{p}$ $<0,001$ ) (Tabelas 2 e 3 ). 
Tabela 2. Evolução temporal de peso, altura, IMC, I0 e D0 lombar de 35 pacientes com transtorno alimentar

\begin{tabular}{lccc}
\hline & Inicial & $\mathbf{6}$ meses & $\mathbf{1}$ ano \\
\hline Escore Z peso & $-1,2$ & $-0,64$ & $-0,56$ \\
& $(-1,85-0,59)$ & $(-1,29-0,02)$ & $(-1,24-0,07)$ \\
Escore Z altura & $-0,45$ & $-0,41$ & $-0,34$ \\
& $(-1,43-0,48)$ & $(-1,38-0,51)$ & $(-1,27-0,53)$ \\
Escore Z IMC & $-1,24$ & $-0,47$ & $-0,38$ \\
& $(-1,97-0,54)$ & $(-1,27-0,8)$ & $(-1,21-0,39)$ \\
Idade óssea, anos & $15,37 \pm 1,78$ & - & $16,42 \pm 1,51$ \\
DO lombar (escore Z) & $-0,45 \pm 1,21$ & $-0,52 \pm 1,21$ & $-0,49 \pm 1,16$ \\
\hline
\end{tabular}

Tabela 3. Comparação das médias do peso, altura, IMC, 10 e D0 lombar de 35 pacientes com transtorno alimentar no início do estudo, após 6 meses e 1 ano de tratamento

\begin{tabular}{lccc}
\hline & T0 x T6m & T0 x T1a & T6m X T1a \\
\hline Peso (escore Z) & $p<0,001$ & $p<0,001$ & $p<0,001$ \\
Altura (escore Z) & $p<0,001$ & $p<0,001$ & $\mathrm{p} \mathrm{0,016}$ \\
IMC (escore Z) & $\mathrm{p}<0,001$ & $\mathrm{p}<0,001$ & $\mathrm{p}<0,001$ \\
Idade óssea, anos & - & $\mathrm{p}<0,001$ & - \\
D0 lombar, DP (escore Z) & $\mathrm{p} \mathrm{0,494}$ & $\mathrm{p} \mathrm{0,765}$ & $\mathrm{p} \mathrm{0,3}$ \\
\hline
\end{tabular}

T0: início do estudo; T6m: após 6 meses; T1a: após 1 ano.

A média do escore $\mathrm{Z}$ de altura no início do tratamento foi de $-0,45(-1,43-0,48)$, após seis meses de tratamento, de $-0,41(-1,38-0,51)$ e após um ano de tratamento, de -0,34 (-1,27-0,53). A comparação das médias do escore $\mathrm{Z}$ de altura entre tempo inicial e após seis meses evidenciou valor de $\mathrm{p}<0,001$. A comparação das médias do escore $\mathrm{Z}$ de altura entre tempo inicial $\mathrm{e}$ após um ano evidenciou valor de $\mathrm{p}<0,001$, e a comparação das médias da altura entre seis meses e um ano evidenciou valor de p de 0,016 (Tabelas 2 e 3 ).

A média do escore $\mathrm{Z}$ de IMC no início do tratamento foi de $-1,24(-1,97-0,54)$, após seis meses de tratamento, de $-0,47(-1,27-0,8)$ e após um ano de tratamento, de-0,38 (-1,21-0,39). A comparação das médias do escore $\mathrm{Z}$ de IMC entre tempo inicial e após seis meses evidenciou valor de $\mathrm{p}<0,001$. A comparação das médias do escore $\mathrm{Z}$ de IMC entre tempo inicial e após um ano evidenciou valor de $\mathrm{p}<0,001$, e a comparação das médias do escore $\mathrm{Z}$ de IMC entre seis meses e um ano evidenciou valor de $\mathrm{p}$ de 0,001 (Tabelas 2 e 3 ).

A média da idade óssea no início do tratamento foi de $15,37 \pm 1,78$ anos e após um ano de tratamento, de $16,42 \pm 1,51$ anos. A comparação das médias da idade óssea entre tempo inicial e após um ano evidenciou valor de $\mathrm{p}<0,001$ (Tabelas 2 e 3 ).
A média do escore $\mathrm{Z}$ da densitometria óssea lombar (Ll-L4) no início do tratamento foi de $-0,45 \pm 1,21$ desvio-padrão (DP), após seis meses de tratamento, de $-0,52 \pm 1,21$ DP e após um ano de tratamento, de $-0,49 \pm 1,16$ DP. A comparação das médias do escore $\mathrm{Z}$ da densitometria óssea entre tempo inicial e após seis meses evidenciou valor de $\mathrm{p}$ de 0,494 . A comparação das médias do escore $\mathrm{Z}$ da densitometria óssea entre tempo inicial e após um ano evidenciou valor de $\mathrm{p}$ de 0,765 e a comparação das médias entre seis meses e um ano evidenciou valor de p de 0,3 (Tabelas 2 e 3 ).

Inicialmente quatro pacientes apresentavam DO lombar Ll-L4, com escore $\mathrm{Z}<-2$ DP $(11,4 \%)$, e essa proporção diminuiu para duas $(5,7 \%)$ após seis meses e um ano de tratamento.

\section{DISCUSSÃO}

Houve aumento significativo do peso, da altura e do IMC das pacientes quando comparados os valores iniciais com os valores com seis meses e um ano de tratamento.

Nosso estudo mostrou boa evolução nutricional tanto das pacientes com AN como das meninas com TANE, com aumento do IMC em 96,4\% dos casos.

Houve progressão da idade óssea e $70 \%$ das adolescentes com amenorreia secundária restabeleceram os ciclos menstruais durante o primeiro ano de tratamento, o que evidencia que houve satisfatória recuperação nutricional com restabelecimento do eixo hipotálamo-hipófise-gônadas.

O retorno dos ciclos menstruais é um indicador de saúde biológica, e a recuperação nutricional exerce efeito anabólico sobre os ossos.

No entanto, não houve diferença significativa do escore $\mathrm{Z}$ da densitometria óssea lombar ao longo do acompanhamento. As médias do escore $\mathrm{Z}$ da DMO da coluna lombar eram normais no início e ao longo do seguimento, pois nos três tempos de avaliação foram acima de -2 .

A AN tem evolução crônica, visto que na maioria dos casos menos da metade das pacientes volta ao peso normal e mantém a função menstrual regular após longos períodos de seguimento (19). A desnutrição causa baixa DMO em mulheres jovens com AN. A rápida perda de massa óssea que ocorre nos primeiros seis meses da doença é bem documentada em mulheres anoréxicas adultas $(20,21)$. No entanto, poucos estudos avaliaram a massa óssea em adolescentes com AN e TANE. 
Como a adolescência é um período crítico para acúmulo mineral ósseo, déficits que ocorrem nessa fase da vida podem ser permanentes, o que aumenta a incidência de osteoporose e consequentemente o risco de fraturas espontâneas na vida adulta.

Alguns autores sugerem que pode ocorrer perda óssea irreversível com o início do TA antes ou durante a adolescência. Mulheres adultas com o início da AN na adolescência tiveram menor massa óssea do que aquelas com o início da AN na vida adulta. O início da AN na adolescência tem impacto mais negativo sobre a DMO do que o início da doença na vida adulta (22).

A grande maioria dos autores relata DMO persistentemente baixa em adolescentes com AN apesar da recuperação nutricional e do ganho de peso. Um estudo longitudinal e prospectivo com adolescentes anoréxicas demonstrou que houve redução da DMO nos primeiros seis meses de evolução e discreta melhora da massa óssea após 24 meses de evolução (23). Um estudo com seguimento por um período de dois anos indicou que uma deficiência residual na massa óssea pode persistir mesmo após a recuperação do peso (24). Tais estudos demonstram que o tempo de evolução é um fator determinante e crucial para a recuperação da massa óssea, portanto a idade ao início do transtorno alimentar, a duração do TA e consequentemente a duração da desnutrição e da amenorreia estão correlacionados significantemente com a redução da densidade óssea.

O ganho de peso e a normalização dos ciclos menstruais são objetivos centrais no tratamento da $\mathrm{AN}$ e fundamentais para recuperação da massa óssea. O achado de a média do escore $\mathrm{Z}$ da DMO lombar estar dentro do normal no início e após seis meses e um ano de tratamento e de não haver diferença significativa ao longo do seguimento era esperado.

Muitas pacientes ainda estavam em fase de crescimento, portanto ainda não atingiram o pico de massa óssea. Inicialmente quatro pacientes apresentavam DMO lombar L1-L4 com escore $\mathrm{Z}<-2$ DP. E, dessas, duas apresentaram recuperação da DMO após seis meses de tratamento e uma paciente apresentou piora significativa da DMO ao longo do seguimento, apesar de ganho de peso satisfatório e regularização dos ciclos menstruais. Recuperar massa óssea é um processo mais demorado do que normalizar o eixo hipotalâmico-hipofisário-gonadal (HHG) (25).

$\mathrm{Na}$ anorexia nervosa, mesmo após um ganho de peso satisfatório, com normalização do IMC e restauração dos ciclos menstruais, há ainda aumento da reab- sorção óssea, que muitas vezes não é compensada pela normalização da formação óssea. Portanto, a DMO não deve ser utilizada como critério único de recuperação nutricional em casos de TA.

O presente estudo tem algumas limitações. O tamanho da amostra é pequeno, o que pode limitar a representatividade dos dados.

O curto período de observação também é um fator limitante, visto que a AN é uma doença crônica e o período de desnutrição na $\mathrm{AN}$ é frequentemente maior do que um ano. Talvez se o acompanhamento das pacientes fosse por um período superior a um ano, teríamos resultados diferentes.

Declaração: os autores declaram não haver conflitos de interesse científico neste estudo.

\section{REFERÊNCIAS}

1. Kaplan HI, Sadock I, et al. Eating Disorders. In: Kaplan HI, Sadock I, et al. Synopsis of psychiatry: behavioral sciences and clinical psychiatry, Seventh Edition, Williams and Wilkins, 1998:720-7.

2. Abreu CN, Cordás TA. Transtornos alimentares: classificação e diagnóstico. Rev Psiquiatr Clin. 2004;31(4):154-7.

3. Eating Disorders. In: American Psychiatric Association: Diagnostic and Statistical Manual of Mental Disorders, Fourth Edition, Washington, DC, American Psychiatric Association, 1994:539-45.

4. American Psychiatric Association Practice Guidelines for the Treatment of Psychiatric Disorders, Compendium 2006. In: Yager J, Devlin MJ, Halmi KA, Herzog DB, Mitchell JE, Powers P, Zerbe KJ. Guidelines for theTreatment of Eating Disorders, Third Edition by American Psychiatric Publishing Inc., Washington D.C.

5. Machado P, Machado BC, Gonçalves S, Hoek HW. The prevalence of eating disorders not otherwise specified. Int $\mathrm{J}$ Eat Disord. 2007;40(1):212-17.

6. Lock J, Bravender T, Bryant-Waugh R, Herzog D, Katzman D, Kreipe RD, et al. Workgroup for Classification of Eating Disorders in Children and Adolescents (WCEDCA). Classification of child and adolescent eating disturbances. Int J Eat Disord. 2007;40:117-22.

7. Mehler PS, Cleary BS, Gaudiani JL. Osteoporosis in anorexia nervosa. Eat Disord. 2011;19(2):194-202.

8. Stone M, Briody J, Kohn MR, Clarke S, Madden S, Cowell CT. Bone changes in adolescent girls with anorexia nervosa. J Adolesc Health. 2006;39:835-41.

9. Canhão $H$, Fonseca JE, Queiroz MV. Diagnóstico e terapêutica da osteoporose na idade pediátrica. Acta Med Port. 2004;17:385-90.

10. Gordon CM, Bachrach LK, Carpenter TO, Crabtree N, El-Hajj Fuleihan G, Kutilek S, et al. Dual energy X-ray absorptiometry interpretation and reporting in children and adolescents: the 2007 ISCD Pediatric Official Positions. J Clin Densitom. 2008;11(1):43-58.

11. Brandão CMA, Camargos BM, Zerbini CA, Plapler PG, Mendonça LMC, Albergaria BH, et al. Posições oficiais 2008 da Sociedade Brasileira de Densitometria Clínica (SBDens). Arq Bras Endocrinol Metab. 2009;53(1):107-12.

12. Do Carmo I, Mascarenhas M. A study of bone density change in patients with anorexia nervosa. Eur Eat Disorders Rev. 2007;15:457-62.

13. Misra M, Klibanski A. Anorexia nervosa and osteoporosis. Rev Endocr Metab Disord. 2006;7:91-9. 
14. Legroux-Gerot I, Vignau J, Dherbomez M, Collier F, Marchandise $X$, Duquesnoy, et al. Evaluation of bone loss and its mechanisms in anorexia nervosa. CalcifTissue Int. 2007;81:174-82.

15. Ogden CL, Kuczmarski RJ, Flegal KM, Mei Z, Guo S, Wei R, et al. Centers for Disease Control and Prevention, 2000. Growth Charts for the United States: improvements to National Center for Health Statistics Version. Pediatrics. 2002;109:45-60.

16. Peter VV, DrizdTA, Johnson CL, Reed RB, Roche AF. NCHS Growth Curves for Children Birth to 18 years. Developed by the National center for Health Statistics in collaboration with the National center for Chronic Disease Prevention and Health promotion (2000). Disponível em: http://www.cdc.gov/growthcharts.

17. Greulich WW, Pyle SI. The rationale and technique of assessing the developmental status of children from roentgenograms of hand and wrist. In: Radiographic Atlas of Skeletal Development of the Hand and Wrist, 2a ed. 1993, Stanford University Press; Stanford, California.

18. Tanner JM, Whitehouse RH, Cameron N, MarshallWA, Healy MJR, Goldstein NH. Assessment of skeletal maturity and prediction of adult height (TW3 method). 3rd ed. London: W.B. Saunders; 2001.

19. Horst-Sikorska W, Ignaszak-Szczepaniak M. The role of anorexia nervosa in secondary osteoporosis development with the risk for low energy fractures. Endokrynol Pol. 2011;62(1):45-7.
20. Schulze UM, Schuler S, Schlamp D, Schneider P, Mehler-Wex C. Bone mineral density in partially recovery early onset anorexic patients - a follow-up investigation. Child Adolesc Psychiatry Ment Health. 2010;8:4-20.

21. Halvorsen I, Platou D, Høiseth A. Bone mass eight years after treatment for adolescent-onset anorexia nervosa. Eur Eat Disord Rev. 2012;20(5):386-92.

22. Misra M. Long-term skeletal effects of eating disorders with onset in adolescence. Ann NY Acad Sci. 2008;1135:212-8.

23. Mika C, Holtkamp K, Heer M, Günther RW, Herpertz-Dahlmann B. A 2-year prospective study of bone metabolism and bone mineral density in adolescents with anorexia nervosa. J Neural Transm. 2007;114(12):1611-8.

24. Milos G, Spindler A, Ruegsegger P, Hasler G, Schnyder U, Laib $A$, et al. Does weight gain induce cortical and trabecular bone regain in anorexia nervosa? A two-year prospective study. Bone. 2007;41:869-74.

25. Misra M, Klibanski A. The neuroendocrine basis of anorexia nervosa and its impact on bone metabolism. Neuroendocrinology. 2011;93(2):65-73. 